\title{
SPECIAL VALUES OF THE RIEMANN ZETA FUNCTION CAPTURE ALL REAL NUMBERS
}

\author{
EMRE ALKAN
}

(Communicated by Ken Ono)

Abstract. It is shown that the set of odd values

$$
\{\zeta(3), \zeta(5), \ldots, \zeta(2 k+1), \ldots\}
$$

of the Riemann zeta function is rich enough to capture real numbers in an approximation aspect. Precisely, we prove that any real number can be strongly approximated by certain linear combinations of these odd values, where the coefficients belonging to these combinations are universal in the sense of being independent of $\zeta(n)$ for all integers $n \geq 2$. This approximation property is reminiscent of the classical Diophantine approximation of Liouville numbers by rationals.

\section{INTRODUCTION}

The Riemann zeta function is defined, for $\mathfrak{R}(s)>1$, as

$$
\zeta(s)=\sum_{n=1}^{\infty} \frac{1}{n^{s}} .
$$

Besides being a central actor in the distribution of prime numbers through the location of its nontrivial zeros, the Riemann zeta function continues to be a rich source of inspiration in complex analysis and arithmetic with the study of its special values. Euler's classical formula on even values, namely

$$
\zeta(2 m)=(-1)^{m-1} \frac{(2 \pi)^{2 m}}{2(2 m) !} B_{2 m}
$$

where $B_{2 m}$ are the (nonvanishing) Bernoulli numbers for $m \geq 1$, surprisingly appears in some energy calculations belonging to quantum mechanics and in Bose condensation phenomena of statistical quantum mechanics (see p. 38 and p. 243 of [20]). A fruitful approach builds on the study of generating functions such as

$$
\sum_{n=1}^{\infty} \chi(n) e^{-n x}=\sum_{k=0}^{\infty} \frac{L(-k, \chi)(-x)^{k}}{k !}
$$

for $0<x<2 \pi / q$, where

$$
L(s, \chi)=\sum_{n=1}^{\infty} \frac{\chi(n)}{n^{s}}
$$

Received by the editors April 21, 2014.

2010 Mathematics Subject Classification. Primary 11M06, 41A50, 42A16.

Key words and phrases. Riemann zeta function, special values, odd values, even values, approximation property. 
is the $L$-function for $\mathfrak{R}(s)>1$ corresponding to the Dirichlet character $\chi$ modulo q. Improving on this approach, Andrews, Jiménez-Urroz and Ono [9] discovered many striking generating function identities concerning the special values of Hecke $L$-functions. Coogan and Ono [18] studied the arithmetic of values of Hurwitz zeta functions in the context of hypergeometric series. Combinatorial connections and relations between values of the hyperharmonic zeta function and the Hurwitz zeta function were nicely treated by Mezö and Dil [25. The author [1]-5] investigated other significant algebraic, statistical and combinatorial properties of values of Dirichlet $L$-functions. Motivated by some trigonometric series transformations of Ramanujan (see [26] and p. 288 of [14]), the author [6] found new rapidly converging series representations of special values of all Dirichlet $L$-functions. Specifically, it is shown for $0 \leq z<2 \pi$ that

$$
\begin{aligned}
G=\frac{1}{16} \sum_{n=0}^{\infty}( & \left.\frac{e^{-\frac{z}{4}}}{\left(n+\frac{1}{4}\right)^{2}}-\frac{e^{-\frac{3 z}{4}}}{\left(n+\frac{3}{4}\right)^{2}}+\frac{z e^{-\frac{z}{4}}}{n+\frac{1}{4}}-\frac{z e^{-\frac{3 z}{4}}}{n+\frac{3}{4}}\right) e^{-n z} \\
& +\left(\sum_{v=1}^{4} B_{n}\left(1-\frac{v}{q}\right)\right) \frac{z^{n+1}}{(n+1) !},
\end{aligned}
$$

where $G$ is Catalan's constant and $B_{n}(x)$ is the Bernoulli polynomial of order $n$ together with the series

$$
\zeta(m)=\sum_{n=1}^{\infty}\left(\sum_{k=0}^{m-1} \frac{1}{n^{m-k} k !}\right) e^{-n}+\frac{B_{n-1}}{(m-1) !(n-1) !(n+m-2)},
$$

for $m \geq 2$. This latter series representation has the advantage of giving a unified treatment to even and odd values of $\zeta(s)$. For other types of rapidly converging series for these values, we urge the reader to look at the works of Berndt [12, 13], especially Chap. 14 of [15], Kanemitsu, Kumagai and Yoshimoto [22, Batır [10], [11] and Vepštas [27. Concerning arithmetic nature of special values, we know that $\zeta(2 m)$ is transcendental as a result of Euler's formula and it is a plausible conjecture that this is so for $\zeta(2 m+1)$ as well. Approximation type results using values of Dirichlet series in the complex plane have a long history of research going back to the classical works of Bohr and Courant [16], [17. They proved, among other things, the surprising fact that the set of values

$$
\{\zeta(\sigma+i t): t \in \mathbb{R}\}
$$

is everywhere dense in the plane for any fixed $\sigma \in\left(\frac{1}{2}, 1\right)$. For a detailed account of recent results on this topic, we refer to [23]. In this connection, the author [4] showed that weighted averages of Gauss sums can be well approximated by $\mathbb{Q}$-linear combinations of special values of $L$-functions in which coefficients of the combinations are independent of the character but its parity. Let us also mention that functional independence of general families of $L$-functions (including the ones that are associated to cusp forms) was shown by Zaharescu and Zaki [28] using a purely algebraic method.

The purpose of this paper is to focus on a new type of density result on the real line satisfied by special values of the Riemann zeta function. Our result below shows that the set of odd values is rich enough to capture real numbers in an approximation aspect, namely that all real numbers can be strongly approximated by using certain linear combinations of odd values. The coefficients appearing in 
these combinations turn out to be universal in the sense of being independent of $\zeta(n)$ for all $n \geq 2$. Moreover, the resulting error tends to zero as the number of terms in the combinations tends to infinity and this is analogous to what happens in a typical Diophantine approximation result, where the error tends to zero as the height of the approximating number tends to infinity. To be more precise, let $2 \leq n_{1}<n_{2}<\cdots<n_{k}<\ldots$ be a sequence of integers. Then we say that the set of values

$$
\left\{\zeta\left(n_{k}\right): k \geq 1\right\}
$$

has the approximation property, if for any given real number $\alpha$ and $\epsilon>0$, there is an integer $r$ tending to infinity as $\epsilon$ tending to zero and a linear combination of the form

$$
\mathcal{L}=\sum_{j=1}^{r} c_{j} \zeta\left(n_{j}\right)
$$

whose coefficients are all nonzero, depend only on $\alpha$ and $\epsilon$ but not on the values $\zeta(n), n \geq 2$, satisfying

$$
|\alpha-\mathcal{L}|<\epsilon .
$$

Existence of sets of special values with the approximation property is far from obvious. Indeed we show that (see Theorem 1) the approximation property holds for the set of odd values in a stronger form. Furthermore, if $\mathcal{A}$ and $\mathcal{B}$ are disjoint sets of special values both having the approximation property, then it is easy to see that $\mathcal{A} \cup \mathcal{B}$ also has the approximation property. Our proof of Theorem 1 is delicate and it can be modified only to give the additional result that the set of even values has the approximation property as well. Consequently, one obtains that

$$
\{\zeta(2), \zeta(3), \ldots, \zeta(n), \ldots\}
$$

has the approximation property. However, classifying all sets of values with the approximation property remains as an interesting open problem. In particular, is it true that any infinite set of values as in (1.1) has the approximation property?

If $F$ is a nonempty set of real numbers, then let us write an $F$-linear combination of real numbers $x_{1}, \ldots, x_{n}$ as

$$
\sum_{k=1}^{n} F x_{k}
$$

unless it is necessary to distinguish the coefficients of the combination. We denote by

$$
\mathbb{Q}\left[\pi^{s}: 1 \leq s \leq n\right]
$$

the $\mathbb{Q}$-vector space with basis $\pi^{s}$ for $1 \leq s \leq n$. The statement of our promised theorem is now as follows.

Theorem 1. Let $\alpha$ be a real number and let $q$ be a positive integer. For any given integer $n \geq 3$, there exists an integer $r \leq n$ tending to infinity with $n$, a sequence of rational polynomials $s_{r}(x)=a_{0}+a_{1} x+\cdots+a_{r} x^{r}$ of degree $r$ converging in $\mathcal{C}^{\infty}[0,2 \pi]$ under the supremum metric with coefficients depending only on $\alpha, n$ and $q$, satisfying

$$
\left|\alpha-\sum_{k=1}^{[r / 2]} c_{k} \zeta(2 k+1)\right| \ll_{\alpha, q} \frac{1}{n^{q}}
$$


when $r \geq 2$, where

$$
c_{k}:=\sum_{m=2 k}^{r}(-1)^{k} \frac{m !(2 \pi)^{m-2 k+1} a_{m}}{(m-2 k+1) !} \neq 0
$$

for all $1 \leq k \leq[r / 2]$.

Clearly, $c_{k} \in \mathbb{Q}\left[\pi^{s}: 1 \leq s \leq r-2 k+1\right]$ and the set of numbers of the form

$$
\sum_{k=1}^{[r / 2]} \mathbb{Q}\left[\pi^{s}: 1 \leq s \leq r-2 k+1\right] \zeta(2 k+1)
$$

for $r \geq 2$ is countable. Thus we conclude from the above theorem that for almost all real numbers $\alpha$ (in the sense of Lebesgue measure),

$$
0<\left|\alpha-\sum_{k=1}^{[r / 2]} c_{k} \zeta(2 k+1)\right| \ll_{\alpha, q} \frac{1}{n^{q}} .
$$

This is reminiscent of the classical Diophantine approximation of Liouville numbers by rational numbers. Recall that a real number $\alpha$ is a Liouville number, if for any integer $q \geq 1$, there exists a rational number $\frac{m}{n}$ with $n>1$ satisfying

$$
0<\left|\alpha-\frac{m}{n}\right|<\frac{1}{n^{q}} .
$$

Historically, Liouville numbers were the first examples of transcendental numbers and belong to a larger family called $U$-numbers according to Mahler's classification (see 24]). Concerning this, the author 8 recently discovered new families of transcendental numbers that are not $U$-numbers by studying integral representations involving carefully selected rational functions whose evaluations lead to specific linear combinations with algebraic coefficients of $\pi$ and Baker periods. Let us remark that analogs of Theorem 1 can be obtained for special values of other Dirichlet series. About this, 7] contains further approximations of reals using values of the harmonic zeta function defined by

$$
\zeta_{H}(s)=\sum_{n=1}^{\infty} \frac{H_{n}}{n^{s}}
$$

for $\mathfrak{R}(s)>1$, where $H_{n}=\sum_{k=1}^{n} \frac{1}{k}$ is the $n$-th harmonic number along with results on a class of integrals that arise in physics as a consequence of energy calculations of charged particle systems.

\section{Proof of Theorem 1}

First note that for $|z|=1$ and $z \neq 1$,

$$
\sum_{n=1}^{\infty} \frac{z^{n}}{n}=-\log (1-z)
$$

holds, where the logarithm has its principal value. Taking $z=e^{i t}$ and looking at the real part of both sides of (2.1), one obtains the Fourier cosine series

$$
-\sum_{n=1}^{\infty} \frac{\cos n t}{n}=\log \left(2 \sin \frac{t}{2}\right)
$$


for $0<t<2 \pi$. For $k \geq 1$, we have

$$
\sum_{n=1}^{k} \cos n t=\frac{\sin \frac{k t}{2} \cos \frac{(k+1) t}{2}}{\sin \frac{t}{2}}
$$

Thus if $t \in[\delta, 2 \pi-\delta]$, where $\delta>0$ is fixed but arbitrary, then it follows from (2.3) that

$$
\left|\sum_{n=1}^{k} \cos n t\right|=O_{\delta}(1)
$$

for these values of $t$. Consequently, the partial sums are uniformly bounded in terms of $\delta$ and by Dirichlet's criterion, the Fourier cosine series in (2.2) is uniformly convergent on $[\delta, 2 \pi-\delta]$. Integrating termwise, we see from $(2.2)$ that

$$
-\sum_{n=1}^{\infty} \frac{1}{n} \int_{\delta}^{2 \pi-\delta} t^{m} \cos n t d t=\int_{\delta}^{2 \pi-\delta} t^{m} \log \left(2 \sin \frac{t}{2}\right) d t
$$

for any positive integer $m$. Defining

$$
c_{m}:=\int_{\delta}^{2 \pi-\delta} t^{m} \cos n t d t
$$

one may deduce by partial integration that

$$
\begin{aligned}
c_{m}= & \frac{1}{n}\left((2 \pi-\delta)^{m} \sin n(2 \pi-\delta)-\delta^{m} \sin n \delta\right) \\
& +\frac{m}{n^{2}}\left((2 \pi-\delta)^{m-1} \cos n(2 \pi-\delta)-\delta^{m-1} \cos n \delta\right)-\frac{m(m-1)}{n^{2}} c_{m-2} .
\end{aligned}
$$

Now using (2.5), the left hand side of (2.4) becomes

$$
\begin{aligned}
& -\sum_{n=1}^{\infty} \frac{1}{n^{2}}\left((2 \pi-\delta)^{m} \sin n(2 \pi-\delta)-\delta^{m} \sin n \delta\right) \\
& -m \sum_{n=1}^{\infty} \frac{1}{n^{3}}\left((2 \pi-\delta)^{m-1} \cos n(2 \pi-\delta)-\delta^{m-1} \cos n \delta\right)+m(m-1) \sum_{n=1}^{\infty} \frac{c_{m-2}}{n^{3}}
\end{aligned}
$$

Since all of the series in (2.6) are uniformly convergent in $\delta$, we may let $\delta \rightarrow 0^{+}$ and iterate (2.6) finitely many times (letting $\delta \rightarrow 0^{+}$at each step) to justify that

$$
\sum_{n=1}^{\infty} \frac{t^{m} \cos n t}{n}
$$

can be integrated termwise from 0 to $2 \pi$. Next consider the evaluation

$$
\int_{0}^{2 \pi} t^{m} \cos n t d t=\sum_{k=1}^{[m / 2]}(-1)^{k-1} \frac{m !(2 \pi)^{m-2 k+1}}{(m-2 k+1) ! n^{2 k}}
$$

when $m \geq 2$. Clearly, we have

$$
\lim _{\delta \rightarrow 0^{+}} \int_{\delta}^{2 \pi-\delta} t^{m} \log \left(2 \sin \frac{t}{2}\right) d t=\int_{0}^{2 \pi} t^{m} \log \left(2 \sin \frac{t}{2}\right) d t
$$


since the right hand side of (2.8) is a convergent improper integral. Assembling (2.4), (2.7) and (2.8), one completes the verification of the formula

$$
\sum_{k=1}^{[m / 2]}(-1)^{k} \frac{m !(2 \pi)^{m-2 k+1}}{(m-2 k+1) !} \zeta(2 k+1)=\int_{0}^{2 \pi} t^{m} \log \left(2 \sin \frac{t}{2}\right) d t
$$

when $m \geq 2$. Let $f$ be a $\mathcal{C}^{\infty}$ function defined on $[0,2 \pi]$ that is not a polynomial. Consider the integral

$$
I_{f}=\int_{0}^{2 \pi} f(t) \log \left(2 \sin \frac{t}{2}\right) d t
$$

which is improper at 0 and $2 \pi$. First of all,

$$
\int_{0}^{\pi / 3} f(t) \log \left(2 \sin \frac{t}{2}\right) d t=-\int_{0}^{\infty} f\left(2 \arcsin \frac{e^{-t}}{2}\right) \frac{t e^{-t}}{\sqrt{1-\frac{e^{-2 t}}{4}}} d t
$$

converges since $f$ is bounded. Similarly,

$$
\int_{5 \pi / 3}^{2 \pi} f(t) \log \left(2 \sin \frac{t}{2}\right) d t=\int_{0}^{\pi / 3} f(2 \pi-t) \log \left(2 \sin \frac{t}{2}\right) d t
$$

converges. (2.11) and (2.12) imply the convergence of the integral in (2.10). Thus $I_{f}$ is a real number. For $d \geq 0$, let $V_{d}([0,2 \pi])$ be the vector space of all real polynomials of degree at most $d$ defined on $[0,2 \pi]$. For any continuous function $w$ on $[0,2 \pi]$, define

$$
E_{d}(w):=\inf _{g \in V_{d}([0,2 \pi])}\|w-g\|
$$

where

$$
\|w-g\|=\sup _{x \in[0,2 \pi]}|w(x)-g(x)| .
$$

For $\delta>0$, define the modulus of continuity of $w$ as the quantity

$$
\tau(w, \delta):=\sup _{\substack{|x-y|<\delta \\ x, y \in[0,2 \pi]}}|w(x)-w(y)| .
$$

Assume that $n \geq 3$ is an integer. For any positive integer $q, f^{(q-1)}$ is a continuously differentiable function on $[0,2 \pi]$. Using a classical theorem of Jackson (see Chap. 14 of [19] and the seminal work of Jackson [21] for many other results on functional approximation theory) on the best approximation of smooth functions with polynomials under the supremum metric, we infer that

$$
E_{n}(f)=O_{q}\left(\frac{1}{n^{q-1}} \tau\left(f^{(q-1)}, \frac{1}{n}\right)\right) .
$$

But $f^{(q-1)}$ is clearly a Lipschitz function on $[0,2 \pi]$ and consequently

$$
\tau\left(f^{(q-1)}, \frac{1}{n}\right)=O_{f, q}\left(\frac{1}{n}\right)
$$

follows. Therefore, from (2.13) and (2.14), the estimate

$$
E_{n}(f)=O_{f, q}\left(\frac{1}{n^{q}}\right)
$$


is obtained. Since $\mathbb{Q}$ is dense in $\mathbb{R},(2.15)$ gives the existence of a rational polynomial $s_{r}(x)=a_{0}+a_{1} x+\cdots+a_{r} x^{r}$ of degree $r \leq n$ (so that $a_{r} \neq 0$ ), where the coefficients depend only on $f, n$ and $q$ such that

$$
\left\|f-s_{r}\right\|=\sup _{x \in[0,2 \pi]}\left|f(x)-s_{r}(x)\right| \ll_{f, q} \frac{1}{n^{q}} .
$$

Since $V_{d}([0,2 \pi])$ is a finite dimensional vector subspace of the space of all continuous functions on $[0,2 \pi]$ equipped with the supremum metric, $E_{d}(f)$ is attained by a polynomial of degree at most $d$. But $f$ is not a polynomial so that we have $E_{d}(f)>$ 0 . Thus using (2.16),

$$
\left\|f-s_{r}\right\|<E_{d}(f)
$$

holds when $n$ is large enough. Clearly, (2.17) forces $r>d$. As $d$ is arbitrary, this shows that $r \leq n$ tends to infinity with $n$. Gathering (2.10) and (2.16), one obtains

$$
\begin{aligned}
& \mid I_{f}-\int_{0}^{2 \pi} s_{r}(t) \log \left(2 \sin \frac{t}{2}\right) d t \mid \\
& \leq\left\|f-s_{r}\right\| \int_{0}^{2 \pi}\left|\log \left(2 \sin \frac{t}{2}\right)\right| d t \ll_{f, q} \frac{1}{n^{q}} .
\end{aligned}
$$

From (2.2), we derive (since termwise integration is valid as shown above)

$$
\int_{0}^{2 \pi} \log \left(2 \sin \frac{t}{2}\right) d t=-\sum_{n=1}^{\infty} \frac{1}{n} \int_{0}^{2 \pi} \cos n t d t=0
$$

and

$$
\int_{0}^{2 \pi} t \log \left(2 \sin \frac{t}{2}\right) d t=-\sum_{n=1}^{\infty} \frac{1}{n} \int_{0}^{2 \pi} t \cos n t d t=0 .
$$

Henceforth, using (2.19) and (2.20), one infers that

$$
\int_{0}^{2 \pi} s_{r}(t) \log \left(2 \sin \frac{t}{2}\right) d t=\sum_{m=2}^{r} a_{m} \int_{0}^{2 \pi} t^{m} \log \left(2 \sin \frac{t}{2}\right) d t .
$$

Next assembling (2.9) and (2.21), we see that

$$
\int_{0}^{2 \pi} s_{r}(t) \log \left(2 \sin \frac{t}{2}\right) d t=\sum_{m=2}^{r} \sum_{k=1}^{[m / 2]}(-1)^{k} \frac{m !(2 \pi)^{m-2 k+1} a_{m}}{(m-2 k+1) !} \zeta(2 k+1) .
$$

For $r \geq 2,(2.22)$ implies that

$$
\int_{0}^{2 \pi} s_{r}(t) \log \left(2 \sin \frac{t}{2}\right) d t=\sum_{k=1}^{[r / 2]} c_{k} \zeta(2 k+1)
$$

where $c_{k}$ is given as in the statement of the theorem. The nonvanishing of all $c_{k}$ is a consequence of the fact that $\pi$ is transcendental. Moreover, these coefficients depend only on $f, n$ and $q$. Thus gathering (2.18) and (2.23), one obtains

$$
\left|I_{f}-\sum_{k=1}^{[r / 2]} c_{k} \zeta(2 k+1)\right| \ll_{f, q} \frac{1}{n^{q}} .
$$


To complete the proof, for any given real number $\alpha$, it suffices to construct a nonpolynomial $\mathcal{C}^{\infty}$ function $f_{\alpha}$ on $[0,2 \pi]$ corresponding to $\alpha$ with the property that $\alpha=I_{f_{\alpha}}$. To this end, choose $\delta>0$ such that

$$
\log \left(2 \sin \frac{t}{2}\right) \geq \frac{\log 2}{2}
$$

holds for $t \in[\pi-2 \delta, \pi+2 \delta] \subseteq[0,2 \pi]$. Then consider a $\mathcal{C}^{\infty}$ function $f$ on $[0,2 \pi]$ defined as

$$
f(t)=\left\{\begin{array}{l}
M \text { if } t \in[\pi-\delta, \pi+\delta], \\
0 \text { if } t \in[0, \pi-2 \delta] \cup[\pi+2 \delta, 2 \pi],
\end{array}\right.
$$

where $M>0$ is a constant and $0 \leq f(t) \leq M$ for all $t \in[0,2 \pi]$ (the construction of such a function $f$ is well known). As a result of (2.24) and (2.25), we have

$$
I_{f}=\int_{0}^{2 \pi} f(t) \log \left(2 \sin \frac{t}{2}\right) d t \geq M \delta \log 2 .
$$

Given any number $\alpha>0$, take $M_{1}>0$ large enough such that $M_{1} \delta \log 2>\alpha$. Let $f_{1}$ be the $\mathcal{C}^{\infty}$ function corresponding to $M_{1}$ and constructed similarly as $f$. Moreover, we also have

$$
I_{f} \leq 2 M \pi \log 2 .
$$

Clearly, we can find $M_{2}>0$ small enough such that $2 M_{2} \pi \log 2<\alpha$. Let $f_{2}$ be the $\mathcal{C}^{\infty}$ function corresponding to $M_{2}$. Then $f_{1}$ and $f_{2}$ are not polynomials and belong to the space $\mathcal{C}^{\infty}[0,2 \pi]$. Next we observe that for any $0 \leq \lambda \leq 1$, the function $f_{\lambda}=\lambda f_{1}+(1-\lambda) f_{2}$ is also in $\mathcal{C}^{\infty}[0,2 \pi]$ and is not a polynomial since $f_{\lambda}(t)=\lambda M_{1}+(1-\lambda) M_{2}>0$ for $t \in[\pi-\delta, \pi+\delta]$ and $f_{\lambda}(t)=0$ for $t \in[0, \pi-2 \delta] \cup[\pi+2 \delta, 2 \pi]$. Consider the function $\Phi: \mathcal{C}^{\infty}[0,2 \pi] \rightarrow \mathbb{R}$ defined by

$$
\Phi(g)=\int_{0}^{2 \pi} g(t) \log \left(2 \sin \frac{t}{2}\right) d t
$$

for any $g \in \mathcal{C}^{\infty}[0,2 \pi]$. From $(2.28)$, it is easy to see that

$$
|\Phi(g)-\Phi(h)| \leq K\|g-h\|,
$$

where

$$
K=\int_{0}^{2 \pi}\left|\log \left(2 \sin \frac{t}{2}\right)\right| d t>0 .
$$

Consequently, $\Phi$ is continuous with respect to the supremum metric on $\mathcal{C}^{\infty}[0,2 \pi]$. The set of functions

$$
\mathcal{F}:=\left\{f_{\lambda}: 0 \leq \lambda \leq 1\right\}
$$

is a connected subset of $\mathcal{C}^{\infty}[0,2 \pi]$ since it is path-wise connected (by the definition of $\mathcal{F})$. Thus $\Phi(\mathcal{F})$ is a connected subset of $\mathbb{R}$ containing $\Phi\left(f_{1}\right)$ and $\Phi\left(f_{2}\right)$. But because of (2.26) and (2.27), $\alpha$ is between $\Phi\left(f_{1}\right)$ and $\Phi\left(f_{2}\right)$. It follows that $\alpha \in \Phi(\mathcal{F})$ and this means that there exists a function $f_{\alpha} \in \mathcal{C}^{\infty}[0,2 \pi]$ which is not a polynomial satisfying

$$
\alpha=\int_{0}^{2 \pi} f_{\alpha}(t) \log \left(2 \sin \frac{t}{2}\right) d t .
$$

A similar argument holds for $\alpha<0$ as well by using $-f_{\alpha}$. Lastly, note that

$$
\int_{0}^{2 \pi} \cos \frac{t}{2} \log \left(2 \sin \frac{t}{2}\right) d t=0 .
$$


Clearly, $\cos \frac{t}{2} \in \mathcal{C}^{\infty}[0,2 \pi]$ is not a polynomial and this handles the case $\alpha=0$. It follows that

$$
\left|\alpha-\sum_{k=1}^{[r / 2]} c_{k} \zeta(2 k+1)\right| \ll_{\alpha, q} \frac{1}{n^{q}}
$$

when $r \geq 2$. Here each $c_{k}$ depends only on $\alpha, n$ and $q$. Finally, the sequence of polynomials $s_{r}(x)$ converges to $f_{\alpha}$ in $\mathcal{C}^{\infty}[0,2 \pi]$. Proof of Theorem 1 is now complete.

A close inspection of the above proof reveals that the arguments of special values with the approximation property that are obtainable through our approach form an arithmetic progression of common difference at most two. This is mainly due to the fact that $\sin t$ and $\cos t$ are fundamental solutions of the differential equation $y^{\prime \prime}+y=0$. Since the characteristic polynomial of an $n$-th order homogeneous equation of the form

$$
y^{(n)}+a_{n-1} y^{(n-1)}+\cdots+a_{1} y^{\prime}+a_{0} y=0
$$

with real coefficients always factors into irreducible linear and quadratic factors, the solutions of such an equation would not be useful in producing, via our method, special values with the approximation property whose arguments form an arithmetic progression of common difference at least three. Thus, although capable of producing examples of values with the approximation property, our method has some limitations.

\section{References}

[1] Emre Alkan, On Dirichlet L-functions with periodic coefficients and Eisenstein series, Monatsh. Math. 163 (2011), no. 3, 249-280, DOI 10.1007/s00605-010-0211-2. MR.2805873 (2012e:11151)

[2] Emre Alkan, On the mean square average of special values of L-functions, J. Number Theory 131 (2011), no. 8, 1470-1485, DOI 10.1016/j.jnt.2011.02.013. MR.2793888

[3] Emre Alkan, Values of Dirichlet L-functions, Gauss sums and trigonometric sums, Ramanujan J. 26 (2011), no. 3, 375-398, DOI 10.1007/s11139-010-9292-8. MR2860694

[4] Emre Alkan, On linear combinations of special values of L-functions, Manuscripta Math. 139 (2012), no. 3-4, 473-494, DOI 10.1007/s00229-011-0526-x. MR2974286

[5] Emre Alkan, Averages of values of L-series, Proc. Amer. Math. Soc. 141 (2013), no. 4, 1161-1175, DOI 10.1090/S0002-9939-2012-11506-0. MR3008864

[6] Emre Alkan, Series representations in the spirit of Ramanujan, J. Math. Anal. Appl. 410 (2014), no. 1, 11-26, DOI 10.1016/j.jmaa.2013.08.021. MR.3109816

[7] Emre Alkan, Approximation by special values of harmonic zeta function and logsine integrals, Commun. Number Theory Phys. 7 (2013), no. 3, 515-550, DOI 10.4310/CNTP.2013.v7.n3.a5. MR.3210726

[8] E. Alkan, Series representing transcendental numbers that are not $U$-numbers, Int. J. Number Theory, to appear. doi: 10.1142/S1793042115500487

[9] George E. Andrews, Jorge Jiménez-Urroz, and Ken Ono, q-series identities and values of certain L-functions, Duke Math. J. 108 (2001), no. 3, 395-419, DOI 10.1215/S0012-7094-0110831-4. MR 1838657 (2002e:11055)

[10] Necdet Batir, Integral representations of some series involving $\left(\begin{array}{c}2 k \\ k\end{array}\right)^{-1} k^{-n}$ and some related series, Appl. Math. Comput. 147 (2004), no. 3, 645-667, DOI 10.1016/S0096-3003(02)008020. MR2011078 (2004h:33009)

[11] Necdet Batir, On the series $\sum_{k=1}^{\infty}\left(\begin{array}{c}3 k \\ k\end{array}\right)^{-1} k^{-n} x^{k}$, Proc. Indian Acad. Sci. Math. Sci. 115 (2005), no. 4, 371-381, DOI 10.1007/BF02829799. MR2184197 (2006j:11170)

[12] Bruce C. Berndt, Modular transformations and generalizations of several formulae of Ramanujan, Rocky Mountain J. Math. 7 (1977), no. 1, 147-189. MR0429703 (55 \#2714) 
[13] Bruce C. Berndt, Analytic Eisenstein series, theta-functions, and series relations in the spirit of Ramanujan, J. Reine Angew. Math. 303/304 (1978), 332-365, DOI 10.1515/crll.1978.303304.332. MR514690 (80b:10035)

[14] Bruce C. Berndt, Ramanujan's notebooks. Part I, Springer-Verlag, New York, 1985. With a foreword by S. Chandrasekhar. MR781125 (86c:01062)

[15] Bruce C. Berndt, Ramanujan's notebooks. Part II, Springer-Verlag, New York, 1989. MR.970033 (90b:01039)

[16] H. Bohr, Uber Diophantische Approximationen und ihre Anwendungen auf Dirichlet'sche Reihen, besonders auf die Riemann'sche Zetafunktion, 5. Skand. Mat. Kongr. Helsingfors (1922), 131-154.

[17] H. Bohr, R. Courant, Neue Anwendungen der Theorie der Diophantischen Approximationen auf die Riemannsche Zetafunktion, J. Reine Angew. Math. 144 (1914), 249-274.

[18] Gwynneth H. Coogan and Ken Ono, A q-series identity and the arithmetic of Hurwitz zeta functions, Proc. Amer. Math. Soc. 131 (2003), no. 3, 719-724 (electronic), DOI 10.1090/S0002-9939-02-06649-2. MR.1937408 (2003j:11017)

[19] Kenneth R. Davidson and Allan P. Donsig, Real analysis and applications, Undergraduate Texts in Mathematics, Springer, New York, 2010. Theory in practice. MR2568574 (2010i:26002)

[20] D. J. Griffiths, Introduction to Quantum Mechanics, Second Edition, Pearson, Prentice Hall, 2005.

[21] Dunham Jackson, The theory of approximation, American Mathematical Society Colloquium Publications, vol. 11, American Mathematical Society, Providence, RI, 1994. Reprint of the 1930 original. MR 1451140 (98a:01022)

[22] Shigeru Kanemitsu, Hiroshi Kumagai, and Masami Yoshimoto, On rapidly convergent series expressions for zeta- and L-values, and log sine integrals, Ramanujan J. 5 (2001), no. 1, 91-104, DOI 10.1023/A:1011449413387. MR:1829811(2002c:11102)

[23] A. A. Karatsuba and S. M. Voronin, The Riemann zeta-function, de Gruyter Expositions in Mathematics, vol. 5, Walter de Gruyter \& Co., Berlin, 1992. Translated from the Russian by Neal Koblitz. MR1183467 (93h:11096)

[24] K. Mahler, Zur Approximation der Exponentialfunktionen und des Logarithmus, I, II, J. Reine Angew. Math. 166 (1932), 118-150.

[25] István Mező and Ayhan Dil, Hyperharmonic series involving Hurwitz zeta function, J. Number Theory 130 (2010), no. 2, 360-369, DOI 10.1016/j.jnt.2009.08.005. MR.2564902 (2010k:11040)

[26] S. Ramanujan, On the integral $\int_{0}^{x} \frac{\tan ^{-1} t}{t} d t$, J. Indian Math. Soc. 7 (1915), 93-96.

[27] Linas Vepštas, On Plouffe's Ramanujan identities, Ramanujan J. 27 (2012), no. 3, 387-408, DOI 10.1007/s11139-011-9335-9. MR2901266

[28] Alexandru Zaharescu and Mohammad Zaki, An algebraic independence result for Euler products of finite degree, Proc. Amer. Math. Soc. 137 (2009), no. 4, 1275-1283, DOI 10.1090/S0002-9939-08-09622-6. MR2465649(2010a:11140)

Department of Mathematics, KoÇ University, Rumelifeneri Yolu, 34450, Sariyer, IsTANBUL, TURKEY

E-mail address: ealkan@ku.edu.tr 